\title{
Rheology of Copolymer Solutions. IV. Nonlinear Viscoelasticity of Solutions of an SBS Block Copolymer
}

\author{
Kunihiro OsaKI, Bong-Shik KIM ${ }^{\dagger}$, and Michio KuRATA* \\ Institute for Chemical Research, Kyoto University, \\ Uji, Kyoto 611, Japan.
}

(Received February 8, 1978)

\begin{abstract}
Nonlinear viscoelasticity was investigated for concentrated solutions of a styrene-butadiene block copolymer, having a 4-armed star-branched polybutadiene structure with a polystyrene block on each end. When the solvent was good for both polystyrene and polybutadiene, the viscoelastic behavior was similar to that of homopolymer solutions. In the case of 1-chlorohexadecane, a nonsolvent for polystyrene at temperatures below $25^{\circ} \mathrm{C}$, the viscoelastic behavior of the solutions was quite different: the rate of shear-temperature and -concentration reduction rules were not applicable to the steady shear viscosity, the Cox-Merz empirical rule was not applicable, and the maximum relaxation time decreased with increasing rate of shear. It was inferred that the behavior of the copolymer solutions could be described by using the $\mathrm{BKZ}$ constitutive model. Model calculations performed for a solution in 1chlorohexadecane revealed that the shear stresses for various flow histories were predicted well if the memory function was evaluated from the strain-dependent relaxation modulus. The characteristic behavior of the solutions in 1-chlorohexadecane was attributable to a strainsensitive relaxation mode of a long relaxation time, presumably due to the molecular aggregate formed through temporary cross-links of precipitated polystyrene blocks.

KEY WORDS Block Copolymer / SBS / Solution / Nonlinear Viscoelasticity / BKZ Constitutive Model /
\end{abstract}

The complicated features of nonlinear behavior are undoubtedly the most important part of the rheology of solutions or melts of block copolymers. Pioneering studies, ${ }^{1-7}$ mostly on steady shear viscosity, have revealed for copolymer systems anomalous behavior of a sort that has not been observed for homopolymer systems. Some of the copolymer systems exhibit a marked thixotropy, $i$. e., the viscosity depends on the deformation history and becomes smaller when the sample has been exposed to high shear. In some cases, the temperature -rate of shear reduction rule is not applicable, and in certain other cases, the Cox-Merz empirical law, giving a correspondence between the steady shear viscosity and the dynamic complex viscosity, is not applicable. Unfortunately, these results have been published in fragments in view of the phenome-

${ }^{\dagger}$ Present Address: Department of Industrial Chemistry, College of Engineering, Yeungnam University, Gyongsan, Dae-Dong, Korea.

* To whom reprints should be requested. nological studies of the nonlinear rheology and so are not very useful for finding fundamental relations of rheology or constitutive equations for the block copolymer systems. Recently, much progress has been made in studies of nonlinear viscoelasticity of solutions or melts of homopolymers. One can expect to be able to describe the nonlinear behavior of copolymer systems in a systematic manner by applying the methods developed for homopolymer systems.

In the present study we investigate not only the steady shear viscosity but the stresses for timedependent flow histories. Our plan is to compare the results with typical results obtained for homopolymer solutions and to examine the origin of anomalous behavior of block copolymer systems in terms of the constitutive equation or the material functions included in the constitutive equation. A part of this present study has been published in previous reports. $^{8,9}$ Studies dealing with a similar idea are also in progress at other laboratories. ${ }^{10}$ 
The linear viscoelastic properties of the present samples, which are concentrated solutions of a styrene-butadiene block copolymer, have already been studied. ${ }^{11}$ When the solvent was good for both polystyrene and polybutadiene, the effect of varying the temperature and concentration on viscoelastic quantities was not much different from that for solutions of homopolymers. On the other hand, when the solvent was a precipitant for polystyrene in the temperature range in which measurment were carried out, anomalous behavior was observed. The time-temperature or -concentration reduction rule was not applicable to the linear viscoelastic functions. A broad tail appeared at the long time end of the relaxation spectrum as the temperature decreased or the concentration increased. The ratio $\tau_{1}^{0} / \eta^{0}$ increased with increasing concentration contrary to the known results for homopolymer solutions, where $\tau_{1}{ }^{0}$ is the maximum relaxation time and $\eta^{0}$ is the zero-shear viscosity. The results were tentatively interpreted in terms of molecular aggregates, presumably formed by intermolecular crosslinks due to the hard domains of precipitated polystyrene blocks.

\section{MATERIALS AND METHOD}

The block copolymer solutions studied here were the same as those studied in a preceding paper, where the polymer structure and the results of solution tests were described in detail. ${ }^{11}$ The polymer, Solprene 411 from Phillips Petroleum Company, was a styrene-butadiene block copolymer having a four-armed star-branched polybutadiene structure with a polystyrene block on each end. The number-average molecular weight of the copolymer was 185,000 and the content of styrene was $31.0 \mathrm{wt} \%$. One of the solvents, chlorinated biphenyl (Kaneclor 500 from Kanegafuchi Chemical Industry Co., Ltd.; abbreviated as CB) was a good solvent for both polybutadiene and polystyrene. 1-Chlorohexadecane (Tokyo Chemical Industry Co., Ltd.; cetyl chloride, CC) was a good solvent for polybutadiene but not for polystyrene at low temperatures. Most of the measurements in the present study were performed for solutions in these two typical solvents. Steady shear viscosity was measured for solutions in di(2-ethylhexyl) phthalate (Nakarai Chemicals, Ltd.; dioctyl phthalate, DOP). This solvent was intermediate in nature between $\mathrm{CB}$ and $\mathrm{CC}$, i.e., a good solvent for polybutadiene and a theta solvent for polystyrene. Details of the sample preparation were given in a preceding paper.

The steady shear viscosity $\eta(\dot{\gamma})$, the viscosity growth function $\bar{\eta}(t, \dot{\gamma})$, and the viscosity decay function $\tilde{\eta}(t, \dot{\gamma})$ were measured with a cone-andplate rheometer (Multipurpose Rheometer, Iwamoto Seisakusho Co., Ltd.). These three functions are the ratios of the shear stress to the rate of shear $\dot{\gamma}$ in steady shear flow, at the start of shear flow, and on sudden cessation of the steady shear flow, respectively. Their behavior at the limit of $\dot{\gamma} \rightarrow 0$ has been investigated in a preceding paper. ${ }^{11}$ The strain-dependent relaxation modulus $G(t, \gamma)$ was measured with a cone-and-plate relaxometer, whose details were given earlier. ${ }^{12}$ Here $G(t, \gamma)$ is the ratio of the shear stress to the magnitude of shear $\gamma$ on sudden application of a constant shear strain.

In order to examine the variation of nonlinear behavior with temperature and concentration, we have applied the method of reduced variables to the steady shear viscosity. ${ }^{13}$ This method of reduced variables is applicable when the ratio $\eta(\dot{\gamma}) / \eta^{0}$ obtained at various temperatures $T$ or concentrations $c$ can be made a unique function of $\dot{\gamma} \tau^{\prime}$ by the appropriate choi e of a factor $\tau^{\prime}$. Here $\eta^{0}$ is the zero-shear viscosity and $\tau^{\prime}$ is a function of $T$ and $c$. For many homopolymer solutions, the method of reduced variables is applicable with

$$
\tau^{\prime} \propto \frac{\eta^{0}}{c^{n}}
$$

where $n$ is a constant to be determined experimentally. Typical values of $n$ are 1 for solutions of relatively low molecular weight and concentration and $2 \sim 3$ for highly concentrated solutions. To examine the applicability of the method of reduced variables, we defined a reference state, which was an arbitrary set of temperature and concentration in the range of measurements, and calculated the reduced viscosity

$$
\eta_{r}(\dot{\gamma})=\frac{\eta(\dot{\gamma}) \eta_{0}{ }^{0}}{\eta^{0}}
$$

from the steady shear viscosity obtained at various temperatures and/or concentrations. Then we plotted the reduced viscosity against the reduced rate of shear 
Rheology of Copolymer Solutions. IV.

$$
\dot{\gamma}_{r}=\dot{\gamma}\left(\frac{\eta^{0}}{\eta_{0}^{0}}\right)\left(\frac{c}{c_{0}}\right)^{n}
$$

to see if a single composite curve was obtained with an appropriate choice for the value of $n$. Here $\eta_{0}{ }^{0}$ and $c_{0}$ are the zero-shear viscosity and concentration at the reference state, respectively.

We examined also the applicability of the CoxMerz empirical law ${ }^{14}$ which states that the steady shear viscosity as a function of rate of shear $\dot{\gamma}$ corresponds to the magnitude of the complex viscosity $\left|\eta^{*}(\omega)\right|$ as a function of angular frequency $\omega$ :

$$
\eta(\dot{\gamma})=\left|\eta^{*}(\omega)\right|_{\omega=\dot{\gamma}}
$$

The Cox-Merz empirical law is applicable to almost all the homopolymer solutions ever examined. ${ }^{13}$

To study the time-dependent nonlinear properties of the copolymer solutions, we compared qualitatively the viscoelastic functions with typical results for homopolymer solutions. Extensive studies on polystyrene solutions ${ }^{15-18}$ have revealed that their viscoelastic properties can be described by the strain-dependent constitutive model of Bernstein, Kearsley, and Zapas (BKZ model). ${ }^{19}$ In the present study, we examined the applicability of the $\mathrm{BKZ}$ model to the block copolymer solutions and tried to find characteristics of block copolymer solutions in the constitutive equation approach.

\section{RESULTS AND DISCUSSION}

\section{Steady Shear Viscosity}

Figure 1 shows the steady shear viscosity of the solution of Solprene 411 in three solvents, chlorinated biphenyl $(\mathrm{CB})$, di(2-ethylhexyl) phthalate (DOP), and 1-chlorohexadecane (CC) of approximately the same concentration, $1.8 \times 10^{2} \mathrm{~kg} \mathrm{~m}^{-3}$, at various temperatures. The dashed lines connect the shoulders of the curves where the viscosity begins to decrease with increasing rate of shear. Apparently, shoulders for each solvent lined up on a constant shear stress $(i . e$., viscosity times rate of shear) independent of the temperature as is seen from the $45^{\circ}$ slope of the dashed lines, i. e., $1 \times 10^{2}$ $\mathrm{Pa}, 2 \times 10^{2} \mathrm{~Pa}$, and $2.5 \times 10^{2} \mathrm{~Pa}$ for CC, DOP, and $\mathrm{CB}$, respectively. Thus the decrease in viscosity began at the higher shear stress as solvent became the better solvent for polystyrene. The rate of shear corresponding to the shoulder will be denoted by $\dot{\gamma}_{c}$ for later convenience.

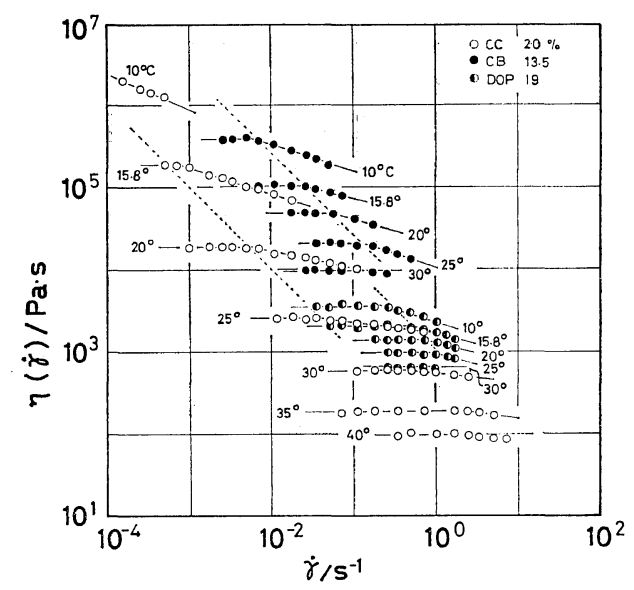

Figure 1. Steady shear viscosity of solutions of Solprene 411 in three solvents. Indicated concentrations in weight per cent correspond to approximately $1.8 \times 10^{2} \mathrm{~kg} \mathrm{~m}^{-3}$. Dashed lines are drawn to indicate roughly the lowest rate of shear where rate dependence of viscosity becomes noticeable.

For the CB solutions, we examined the applicability of the reduced variables method using eq 2 and 3 over the concentration range $0.984 \times 10^{2}-$ $2.74 \times 10^{2} \mathrm{~kg} \mathrm{~m}^{-3}(7-21 \%)$ and the temperature range $10-30^{\circ} \mathrm{C}$. The method of reduced variables was applicable with $n=0.7$ in eq 3 . The temperature and concentration dependence of the factor $\tau^{\prime}$ was thus

$$
\tau^{\prime} \propto \frac{\eta^{0}(T, c)}{c^{0.7}}
$$

for the $\mathrm{CB}$ solutions. It may be noted that this dependence is the same as that of the maximum relaxation time $\tau_{1}{ }^{0}$ estimated from the linear viscoelastic function. ${ }^{11}$ Also, it may be noted that the method of reduced variables with respect to time and concentration was not applicable to linear viscoelastic functions. The steady shear viscosity did not show such delicate variation in shape with varying concentration as the linear viscoelastic functions did; the former is, so to speak, asmoothed-out viscoelastic function as pointed out previously. ${ }^{18,20}$

Figure 2 shows the effect of varying the temperature on the rate dependence of viscosity for a $1.75 \times 10^{2} \mathrm{~kg} \mathrm{~m}^{-3}(20 \%)$ solution of Solprene 411 in CC. The reduced steady shear viscosity $\eta\left(\eta_{0}{ }^{0} / \eta_{0}\right)$ was plotted against the reduced rate of shear $\dot{\gamma}\left(\eta^{0} / \eta_{0}{ }^{0}\right)$, where $\eta_{0}{ }^{0}$ was the zero-shear viscosity at 


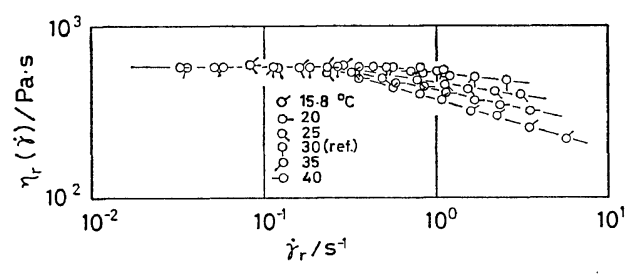

Figure 2. Reduced viscosity $\eta_{r}$ plotted against reduced rate of shear $\dot{\gamma}_{r}$ for $20-\%\left(1.75 \times 10^{2} \mathrm{~kg} \mathrm{~m}^{-3}\right.$ at $20^{\circ} \mathrm{C}$ ) solution of Solprene 411 in 1-chlorohexadecane. Reference temperature is $30^{\circ} \mathrm{C}$.

the reference temperature, $30^{\circ} \mathrm{C}$ in this case. $\mathrm{Ob}$ viously, the reduced points did not superimpose on one another to form a single composite curve: the method of reduced variables was not applicable to the solution. The reduced curve for each temperature was approximated with two straight lines. They intersected at a constant reduced rate of shear independent of the temperature. The slope of the line at the higher rates of shear increased as the temperature decreased.

Applicability of the method of reduced variables with respect to the rate of shear and the concentration was examined on the steady shear viscosity for $15-30 \%\left(1.31 \times 10^{2}-2.65 \times 10^{2} \mathrm{~kg} \mathrm{~m}^{-3}\right.$ at $\left.20^{\circ} \mathrm{C}\right)$ solutions of Solprene 411 in CC. A graph similar to Figure 2 was obtained with $n=-1$ in eq 3; the break points of reduced curves were located at a constant reduced rate of shear independent of concentration and the slope of the reduced curve at high rates of shear increased with increasing concentration. Thus the higher concentration corresponded in effect to the lower temperature. The results on the break points of reduced curves indicate the effect of varying temperature and concentration on the rate of shear $\dot{\gamma}_{c}$ which represents the onset of nonNewtonian behavior of viscosity;

$$
\frac{1}{\dot{\gamma}_{c}} \propto c \eta^{0}(T, c)
$$

As shown previously, ${ }^{11}$ the maximum relaxation time $\tau_{1}{ }^{0}$ for solutions of Solprene 411 in CC varies in proportion to $c \eta^{0}(T, c)$. Thus, eq 6 implies that $1 / \dot{\gamma}_{c}$ is proportional to $\tau_{1}{ }^{0}$ in accord with the wellknown result for homopolymer solutions. ${ }^{13}$ The only difference is that the ratio $1 /\left(\dot{\gamma}_{c} \eta^{0}\right)$ increases with $c$ in eq 6 while it decreases for homopolymers. The specific concentration dependence of $\tau_{1}{ }^{0}$ for the copolymer solutions in $\mathrm{CC}$ was tentatively in-

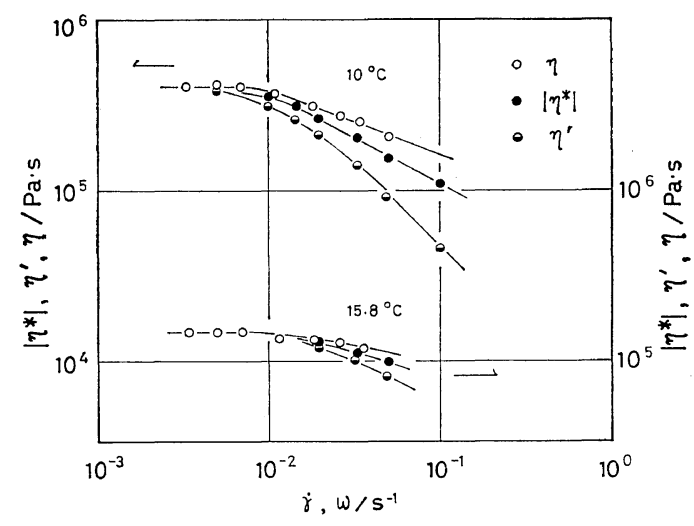

Figure 3. Steady shear viscosity $\eta(\dot{\gamma})$, dynamic viscosity $\eta^{\prime}(\omega)$, and magnitude of complex viscosity $\left|\eta^{*}(\omega)\right|$ for $14-\%\left(1.90 \times 10^{2} \mathrm{~kg} \mathrm{~m}^{-3}\right.$ at $\left.20^{\circ} \mathrm{C}\right)$ solution of Solprene 411 in chlorinated biphenyl. Viscoelastic functions are plotted against respective arguments, rate of shear $\dot{\gamma}$ and angular frequency $\omega$, in the same scale.

terpreted in terms of molecular aggregate formed through intermolecular crosslinks due to the hard domains of precipitated polystyrene blocks. ${ }^{11}$

Figure 3 compares the steady shear viscosity $\eta(\dot{\gamma})$, the dynamic viscosity $\eta^{\prime}(\omega)$, and the magnitude of complex viscosity $\left|\eta^{*}(\omega)\right|$ for a solution of Solprene 411 in chlorinated biphenyl. The viscoelastic functions were plotted against their respective arguments on a common scale. The real part $\eta^{\prime}(\omega)$ and imaginary part $\eta^{\prime \prime}(\omega)$ of the complex viscosity were calculated from the linear viscosity growth function $\bar{\eta}(t)$ with approximation formulae, ${ }^{21}$

$$
\begin{aligned}
\eta^{\prime}(\omega) & \simeq \bar{\eta}(t)-1.08[\bar{\eta}(2.50 t)-\bar{\eta}(1.59 t)] \\
& -\left.0.159[\bar{\eta}(0.40 t)-\bar{\eta}(0.25 t)]\right|_{t=1 / \omega} \\
\eta^{\prime \prime}(\omega) & \simeq 0.470[\bar{\eta}(2 t)-\bar{\eta}(4 t)]+1.715[\bar{\eta}(2 t)-\bar{\eta}(t)] \\
& +\left.0.902[\bar{\eta}(t / 2)-\bar{\eta}(t / 4)]\right|_{t=1 / \omega}
\end{aligned}
$$

Apparently the Cox-Merz empirical law, eq 4, was not applicable. However, the difference between $\eta(\dot{\gamma})$ and $\left|\eta^{*}(\omega)\right|$ was not very large. The critical rate of shear $\dot{\gamma}_{c}$ approximately corresponded to the critical angular frequency $\omega_{c}$ where $\left|\eta^{*}\right|$ began to decrease. The rate of decrease of $\left|\eta^{*}\right|$ was larger than that of $\eta$.

Figure 4 gives a comparison of $\eta(\dot{\gamma}), \eta^{\prime}(\omega)$, and $\left|\eta^{*}(\omega)\right|$ for a solution in CC. Again, the CoxMerz empirical law was not applicable. In the 
Rheology of Copolymer Solutions. IV.

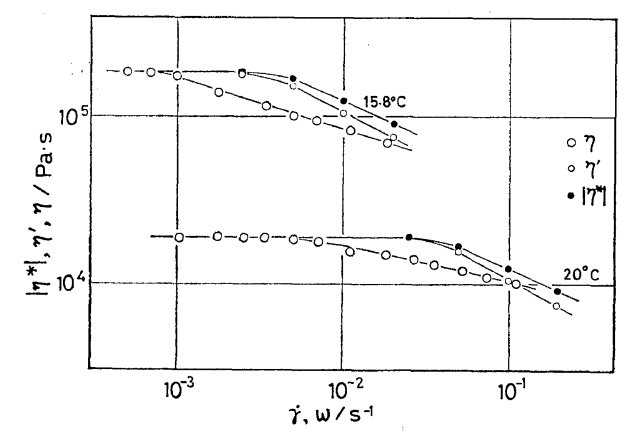

Figure 4. Steady shear viscosity $\eta(\dot{\gamma})$, dynamic viscosity $\eta^{\prime}(\omega)$, and magnitude of complex viscosity $\left|\eta^{*}(\omega)\right|$ for $20-\%\left(1.75 \times 10^{2} \mathrm{~kg} \mathrm{~m}^{-3}\right.$ at $\left.20^{\circ} \mathrm{C}\right)$ solution of Solprene 411 in 1-chlorohexadecane. Viscoelastic functions are plotted against respective arguments, rate of shear $\dot{\gamma}$ and angular frequency $\omega$, in the same scale.

present case, the critical rate of shear $\dot{\gamma}_{c}$ was smaller than the critical angular frequency $\omega_{c}$ by about a factor of 3. Thus the viscosity of the block copolymer solution in $\mathrm{CC}$ began to decrease at a much lower rate of shear than was expected from the dynamic mechanical properties in linear viscoelasticity. ${ }^{13,14}$ The sensitive rate dependence of the viscosity is probably related to a strong strain dependence of the memory function in the constitutive equation as will be discussed later.

\section{Time-Dependent Functions}

Figure 5 shows the viscosity growth function $\bar{\eta}(t, \dot{\gamma})$ for the $13.5 \%\left(1.84 \times 10^{2} \mathrm{~kg} \mathrm{~m}^{-3}\right)$ solution of Solprene 411 in chlorinated biphenyl. At low rates of shear, the function $\bar{\eta}(t, \dot{\gamma})$ monotonously increased with time to approach the steady value

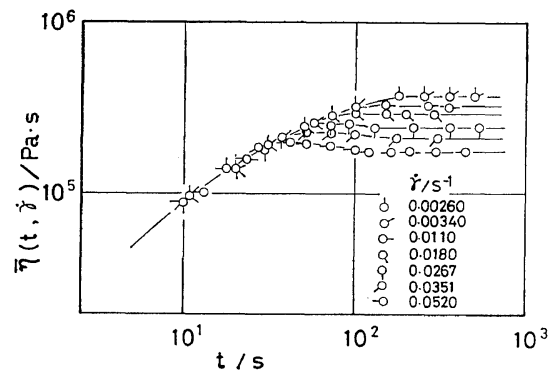

Figure 5. Viscosity growth function for $13.5-\%$ $\left(1.84 \mathrm{~kg} \mathrm{~m}^{-3}\right.$ at $\left.20^{\circ} \mathrm{C}\right)$ solution of Solprene 411 in chlorinated biphenyl. $\eta(\dot{\gamma})$. At high rates of shear, this function first increased with time, passed over a maximum (phenomenon of stress overshoot), and then decreased to approach the steady value. The times at which the function $\bar{\eta}(t, \dot{\gamma})$ took maximum were 90,60 , and $42 \mathrm{~s}$ at rates of shear $2.67 \times 10^{-2}, 3.51 \times$ $10^{-2}$, and $5.20 \times 10^{-2} \mathrm{~s}^{-1}$, respectively. Hence the magnitutes of shear $\gamma_{m}$ applied to the sample before $\bar{\eta}(t, \dot{\gamma})$ became maximum were 2.4, 2.1, and 2.2, respectively. The phenomenon of stress overshoot was observed also at different concentrations and temperatures. The magnitude of shear $\gamma_{m}$ corresponding to the maximum of $\bar{\eta}(t, \dot{\gamma})$ was always in the range $2.0-2.5$ and the value did not vary in a systematic way with varying concentration, temperature, or rate of shear. The value was a little smaller than 2.8-3 obtained for monodisperse polystyrene solutions of various molecular weights and concentrations. ${ }^{18}$

Figure 6 shows the viscosity decay function $\tilde{\eta}(t, \dot{\gamma})$ for the $13.5-\%$ solution of Solprene 411 in chlorinated biphenyl. The function $\tilde{\eta}(t, \dot{\gamma})$ decreased as the rate of shear increased. However, the shape of the curves on the log-log scales was not affected much by the increasing rate of shear. In the range of long times, $t>50 \mathrm{~s}$, the curves were superimposable with one another by parallel shift along the ordinate. Thus the maximum relaxation time was expected to be independent of the rate of shear. Figure 7 shows the plot of $\log \tilde{\eta}(t, \dot{\gamma})$ against $t$ for a few values of $\dot{\gamma}$. In this figure, the data at relatively long times lay on a straight line for each rate of shear and the slope of the line was not affected by the rate of shear. The result in Figure 7 indicated that the viscosity decay function

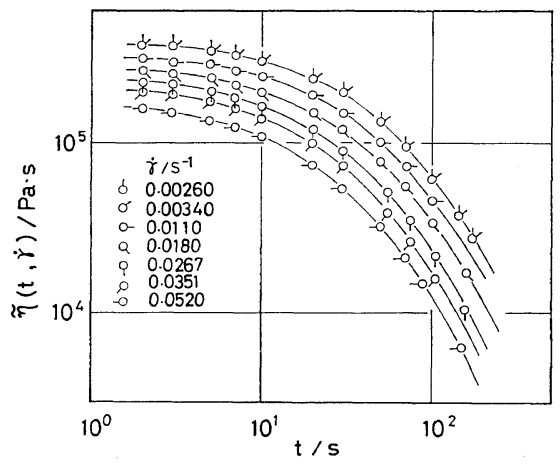

Figure 6. Viscosity decay function for $13.5-\%$ solution of Solprene 411 in chlorinated biphenyl. 


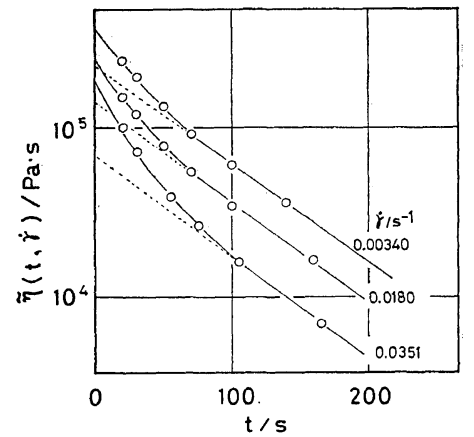

Figure 7. Data of Figure 6 replotted in semilogarithmic scales.

$\tilde{\eta}(t, \dot{\gamma})$ at long times could be approximated by the expression

$$
\tilde{\eta}_{1}(t, \dot{\gamma})=\eta_{1}(\dot{\gamma}) \exp \left(-\frac{t}{\tau_{1}(\dot{\gamma})}\right)
$$

which includes two functions, $\eta_{1}(\dot{\gamma})$ and $\tau_{1}(\dot{\gamma})$, of the rate of shear $\dot{\gamma}$. The relaxation time $\tau_{1}(\dot{\gamma})$ was $75 \mathrm{~s}$ for any rate of shear, and the function $\eta_{1}(\dot{\gamma})$ was $2.40 \times 10^{5}, 1.43 \times 10^{5}$, and $6.90 \times 10^{4} \mathrm{~Pa} \mathrm{~s}$ at rates of shear $3.4 \times 10^{-3}, 1.8 \times 10^{-2}$, and $3.5 \times$ $10^{-2} \mathrm{~s}^{-1}$, respectively, from Figure 7. The independence of the maximum relaxation time from the rate of shear is in accord with the result obtained for polystyrene solutions. ${ }^{17}$ We approximated the function $\eta_{1}(\dot{\gamma})$ by

$$
\eta_{1}(\dot{\gamma})=\frac{\eta_{1}(0)}{\left(1+\alpha \tau_{1}{ }^{0} \dot{\gamma}\right)^{2}}
$$

for later convenience, where $\alpha$ is a constant and $\tau_{1}{ }^{0}$ is the maximum relaxation time at the limit of $\dot{\gamma} \rightarrow 0\left(\tau_{1}{ }^{0}=75 \mathrm{~s}\right.$ in the present case). The value of $\alpha$ obtained from the above figures was 0.42 , which was a little larger than 0.37 obtained for polystyrene solutions. ${ }^{18}$

The results on the functions $\bar{\eta}(t, \dot{\gamma})$ and $\tilde{\eta}(t, \dot{\gamma})$ indicate that the time-dependent nonlinear flow behavior of solutions of Solprene 411 in $\mathrm{CB}$ is quite similar to that of polystyrene solutions. The difference revealed so far is that the values of $\gamma_{m}$ and $\alpha$ are slightly smaller and larger, respectively, than those of polystyrene solutions. ${ }^{16-18}$

Figure 8 shows the viscosity growth function $\bar{\eta}(t, \dot{\gamma})$ for the $20 \%\left(1.75 \times 10^{2} \mathrm{~kg} \mathrm{~m}^{-3}\right)$ solution of Solprene 411 in 1-chlorohexadecane. The function monotonously increased with time at any rate of shear. The phenomenon of stress overshoot

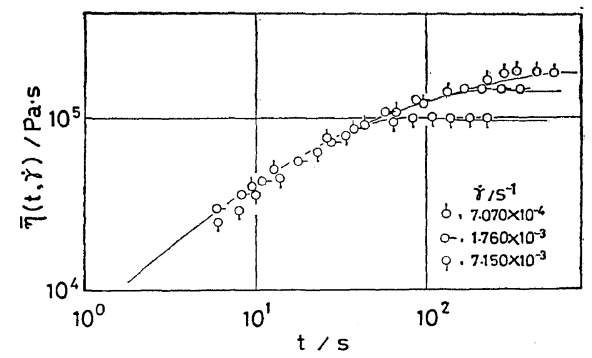

Figure 8. Viscosity growth function for $20-\%(1.75 \times$ $10^{2} \mathrm{~kg} \mathrm{~m}^{-3}$ at $20^{\circ} \mathrm{C}$ ) solution of Solprene 411 in 1chlorohexadecane. Curves represent calculated results due to $\mathrm{BKZ}$ constitutive model.

was not detected at any concentration and temperature investigated.

Figure 9 shows the viscosity decay function $\tilde{\eta}(t, \dot{\gamma})$ for the $20-\%$ solution of Solprene 411 in CC. In contrast with Figure 6 for the solution in $C B$, the shape of curves varied a little with varying the rate of shear. The effect of the shear rate was much greater at long times than at short times. The long time ends of curves could not be superimposed on each other by a parallel shift along the ordinate: the maximum relaxation time was affected by the rate of shear. Figure 10 gives the plot of $\log \tilde{\eta}(t, \dot{\gamma})$ against $t$. The points at relatively long times lay on a straight line at each rate of shear. The slope of the lines increased (in absolute value) as the rate of shear increased. The maximum relaxation time evaluated from the slope decreased by a factor of about 4 as the rate of shear increased from $7.07 \times 10^{-4} \mathrm{~s}^{-1}$ to $7.42 \times 10^{-3} \mathrm{~s}^{-1}$. It

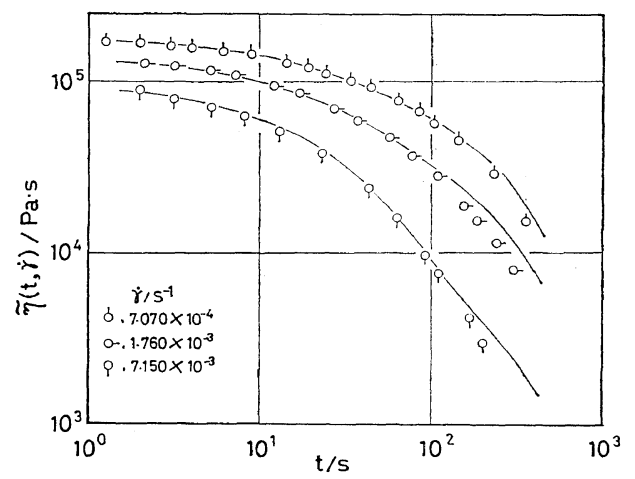

Figure 9. Viscosity decay function for $20-\%$ solution of Solprene 411 in 1-chlorohexadecane. Curves represent calculated results due to $\mathrm{BKZ}$ constitutive model. 


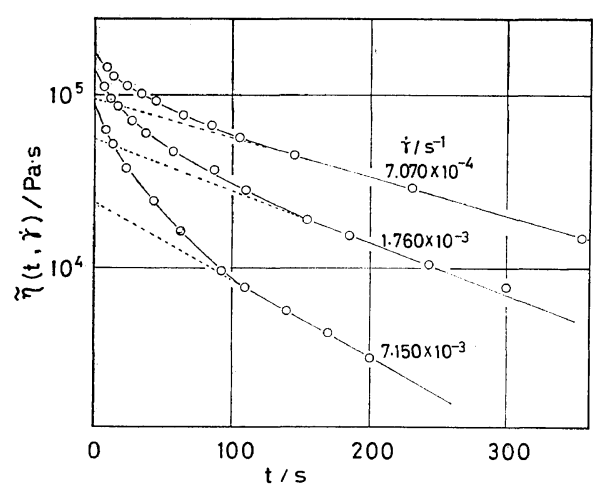

Figure 10. Data of Figure 9 replotted in semilogarithmic scales.

has been reported that relaxation time decreases as the rate of shear increases for homopolymer systems. ${ }^{22}$ However, this describes the overall behavior of the relaxation spectrum since it is based on data at much shorter times compared to the maximum relaxation time. The copolymer solution of the present study is probably the first sample for which the maximum relaxation time is found to be largely affected by the rate of shear.

It may be noted that the variation of curves in Figure 9 with increasing rate of shear is similar to that observed with increasing temperature or decreasing concentration at the limit of $\dot{\gamma} \rightarrow 0 .{ }^{8}$ The three factors, i.e., increasing $\dot{\gamma}$, increasing $T$, and decreasing $c$, may probably have similar effects in destroying the structure of the flow unit in solutions of the copolymer in $\mathrm{CC}$.

\section{Constitutive Equations}

We have revealed in a previous paper $^{18}$ that the shear stresses, in time-dependent shear flows for polystyrene, can be described by the strain-dependent constitutive model proposed by Bernstein, Kearsley, and Zapas (BKZ model). ${ }^{19}$ According to this model, the shear stress $\sigma(t)$, corresponding to any time-dependent shear flow, is given by

$$
\sigma(t)=\int_{-\infty}^{t} \mu\left(t-t^{\prime},\left|\gamma\left(t^{\prime}, t\right)\right|\right) \gamma\left(t^{\prime}, t\right) \mathrm{d} t^{\prime}
$$

where $\gamma\left(t^{\prime}, t\right)$ is the increase in the magnitude of shear in the time interval from $t^{\prime}$ to $t$. The material property is characterized by the memory function $\mu$, which depends on the magnitude of shear $\gamma\left(t^{\prime}, t\right)$ as well as on the time interval $t-t^{\prime}$. The memory function may be evaluated from the strain-dependent relaxation modulus by

$$
\mu(t, \gamma)=-\frac{\partial G(t, \gamma)}{\partial t}
$$

For polystyrene solutions of moderate molecular weight and concentration, the memory function can be approximated by a simple formula

$$
\mu(t, \gamma)=-\frac{\mathrm{d} G(t)}{\mathrm{d} t} \mathrm{e}^{-\alpha \gamma}
$$

where $G(t)$ is the linear relaxation modulus and $\alpha$ is a constant. ${ }^{18}$ The constant $\alpha$ is approximately 0.37 and is independent of molecular weight, concentration, and temperature unless the molecular weight or concentration is very high. Approximately, the same value of $\alpha$ may be applicable to solutions of $\operatorname{poly}(\alpha$-methylstyrene) and polyisobutylene. $^{18,22,23}$ Equations 11 and 13 give

$$
\gamma_{m}=\frac{1}{\alpha}
$$

for the shear strain applied before the viscosity growth function attains maximum. The function $\eta_{1}(\dot{\gamma})$, representing the long time behavior of viscosity decay function, is given by eq 10 .

The rheological behavior of solutions of Solprene 411 in chlorinated biphenyl was similar to that of polystyrene solutions in many respects; for example, the stress overshoot was observed at the start of the steady shear flow and the maximum relaxation time of the stress decay on a sudden cessation of steady shear was not affected by the rate of shear. Equation 13 gives $\gamma_{m}=1 / 0.42=2.4$, which is well in the range of the observed values 2.0-2.5. Thus, the behavior of the copolymer solutions in CB is likely to be described by the BKZ model with the memory function of eq 12 . The value 0.42 for $\alpha$ is a little larger than 0.37 for polystyrene solutions. Thus, the flow properties of the copolymer solution in $\mathrm{CB}$ is slightly more sensitive to the strain than those of polystyrene solutions.

On the other hand, the rheological properties of solutions of Solprene 411 in 1-chlorohexadecane were more complicated and further qualitative comparison with those of polystyrene solutions seemed fruitless. Therefore, we measured the strain-dependent relaxation modulus $G(t, \gamma)$, evalu- 


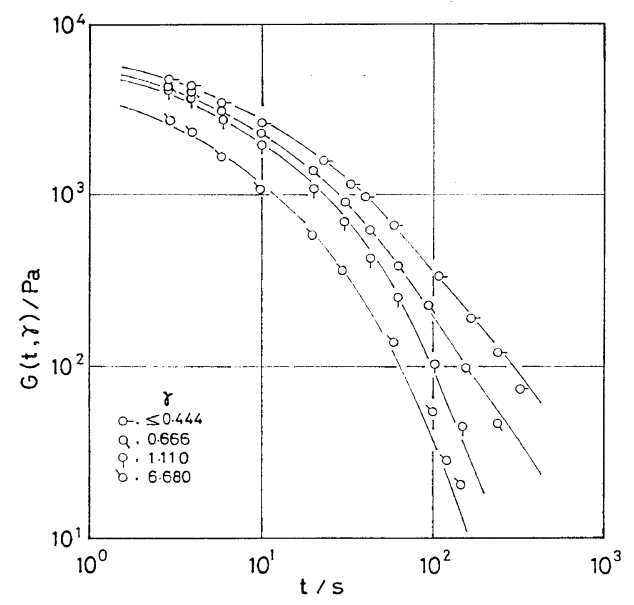

Figure 11. Strain-dependent relaxation modulus for $20-\%$ solution of Solprene 411 in 1-chlorohexadecane. Curves represent calculated results due to approximate formula as given in text (eq 16 and 17 and Table I).

ated the memory function with eq 12 , and calculated the rheological functions $\bar{\eta}(t, \dot{\gamma}), \tilde{\eta}(t, \dot{\gamma})$, and $\eta(\dot{\gamma})$ by eq 11 to make a comparison with experimental results.

The strain-dependent relaxation modulus was measured over the range $0.222 \leq \gamma \leq 6.68$. Figure 11 gives a few examples of the measured results. In the range of small strains, $\gamma \leq 0.444$, the relaxation modulus was not affected by $\gamma$. Thus, the result for $\gamma=0.444$ represents the linear relaxation modulus $G(t)$. At higher shear, the relaxation modulus decreased with increasing shear strain. The relative amount of decrease, i. e., the decrease of $\log G(t, \gamma)$, was larger at long times than at short times. Obviously, it was not possible to express the memory function in a factored form, eq 12, in terms of two functions, one of time and the other of magnitude of shear. Therefore, we assigned the functional form

$$
\begin{gathered}
\mu(t, \gamma)=\sum_{p} \frac{G_{p}{ }^{0}}{\tau_{p}^{0}} f_{p}(\gamma) \mathrm{e}^{-t / \tau_{p}}{ }^{0} \\
\left(\tau_{1}^{0}>\tau_{2}^{0}>\tau_{3}^{0} \cdots\right)
\end{gathered}
$$

to the memory function. Thus, the relaxation modulus can be written as

$$
G(t, \gamma)=\sum_{p} G_{p}{ }^{0} f_{p}(\gamma) \mathrm{e}^{-t / \tau} p^{0}
$$

Here, constants $\tau_{p}{ }^{0}$ and $G_{p}{ }^{0}$ are the $p$-th relaxation time and the relaxation strength, respectively, for
Table I. Relaxation time $\tau_{p}{ }^{0}$ and relaxation strength $G_{p}{ }^{0}$ for $20-\%$ solution of Solprene 411 in 1-chlorohexadecane at $15.8^{\circ} \mathrm{C}$

\begin{tabular}{lrrrrr}
\hline \multicolumn{1}{c}{$p$} & 1 & 2 & 3 & 4 & 5 \\
\hline$\tau_{p}{ }^{0} / s$ & 230 & 35 & 14 & 5.6 & 2.2 \\
$G_{p} / P_{a}$ & 380 & 1800 & 1440 & 1600 & 2000 \\
\hline
\end{tabular}

describing the linear viscoelasticity and the function $f_{p}(\gamma)$ represents the strain-dependence. ${ }^{* 1}$ The parameters $\tau_{p}{ }^{0}$ and $G_{p}{ }^{0}$ were determined by trial and error so that experimental results for the linear relaxation modulus $G(t)$ and the linear stress decay function $\widetilde{\eta}(t)$ could be reproduced. The results are given in Table $\mathrm{I}$. The linear functions $G(t)$ and $\tilde{\eta}(t)$ calculated from the parameters are represented by the uppermost curves in Figures 11 and 9, respectively. Obviously, the calculated values are in close agreement with the observed. The functions $f_{p}(\gamma)$ were then determined by trial and error so that the experimental results for the strain-dependent relaxation modulus $G(t, \gamma)$ could be reproduced. Following results were obtained.

$$
\begin{array}{rlr}
f_{1}(\gamma) & =1 & \gamma<0.5 \\
& =15.66 \mathrm{e}^{-5.6 \gamma}+0.05 \mathrm{e}^{-0.1 \gamma} & \gamma \geq 0.5 \\
f_{2}(\gamma) & =0.3 \mathrm{e}^{-0.7 \gamma}+0.7 \mathrm{e}^{-0.14 \gamma} & \\
f_{3}(\gamma) & =f_{4}(\gamma)=f_{5}(\gamma)=\mathrm{e}^{-0.35 \gamma} &
\end{array}
$$

The relaxation modulus calculated from eq 15 is compared with the experimental values in Figure 11. The agreement was excellent. Thus, we obtained a good set of parameters $\tau_{p}{ }^{0}$ and $G_{p}{ }^{0}$ and functions $f_{p}(\gamma)$, which are suitable for approximating linear viscoelastic functions and the straindependent relaxation modulus.

Using the parameters and functions, we calculated the viscosity growth function $\bar{\eta}(t, \dot{\gamma})$, viscosity decay function $\widetilde{\eta}(t, \dot{\gamma})$, and steady shear viscosity $\eta(\dot{\gamma})$ from eq 11 and 15 . The results are shown by the curves in Figures 8, 9, and 12. The calculated

*1 In view of the result that the maximum relaxation time depends on the rate of shear (see Figure 10), it may be better to assume that the relaxation time depends on the shear strain. We have preferred the form of eq 15 and 16 since, otherwise, the numerical calculations become intolerably complicated and because eq 16 is sufficient to reproduce the data of $G(t, \gamma)$ with a suitable choice of parameters. 
values are in close agreement with those observed in all cases. We may conclude that the BKZ model was suitable for describing the shear stresses in time-dependent shear flows as treated in the present study.

\section{Further Comments}

The result obtained in the preceding section implies that the type of constitutive equation suitable for the copolymer solutions is not different from that for homopolymer solutions. Thus, the characteristic features of the rheology of the copolymer solutions must be deduced from the property of the memory function involved in the constitutive equation. Here, we should like to discuss the property of the memory function for solutions of Solprene 411 in 1-chlorohexadecane.

One of the marked features of the memory function is that the effects of temperature and concentration on one or a few modes related to long relaxation times are much greater than those on other modes of shorter relaxation times. This difference in the effects of temperature and concentration leads to the failure of the method of reduced variables with respect to time-temperature and time-concentration for linear viscoelasticity functions. ${ }^{11}$ The failure of the method of reduced variables with respect to the rate of shear-temperature and rate of shear-concentration for the steady shear viscosity is apparently due to the same origin.

Another characteristic feature of the memory function is that the effect of strain on a few terms of long relaxation times is extremely big. Figure 13 shows the strain-dependent relaxation spectrum $H(\tau, \gamma)$ defined by ${ }^{24}$

$$
G(t, \gamma)=\int_{-\infty}^{\infty} H(\tau, \gamma) \mathrm{e}^{-t / \tau} \mathrm{d} \ln \tau
$$

for the $20-\%$ solution of Solprene 411 in 1-chlorohexadecane. The curves in Figure 13 were calculated from the strain-dependent relaxation modulus $G(t, \gamma)$ with the approximation method of Ferry and Williams. ${ }^{25}$ The relaxation spectrum decreases very rapidly with increasing shear strain at long times; e.g., at $\tau=200 \mathrm{~s}, H(\tau, \gamma)$ decreases by a factor of about 10 while $\gamma$ varies from 0.444 to 1.11. For typical polystyrene solutions, the decrease in $H(\tau, \gamma)$ in the same range of $\gamma$ is only about $30 \%{ }^{16}$ One can easily show that the inapplicability of the Cox-Merz law as shown in Figure

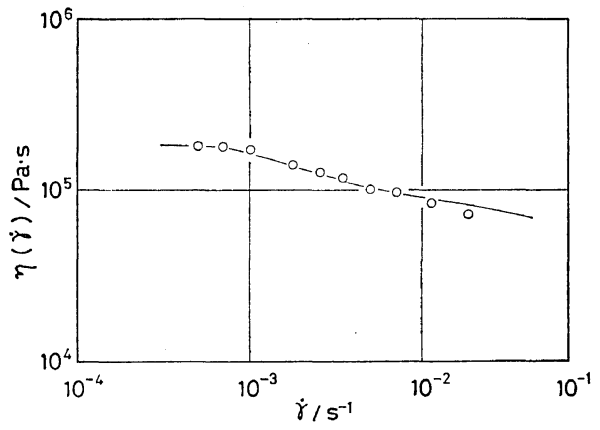

Figure 12. Steady shear viscosity of $20-\%$ solution of Solprene 411 in 1-chlorohexadecane. Curve represent calculated result due to $\mathrm{BKZ}$ constitutive model.

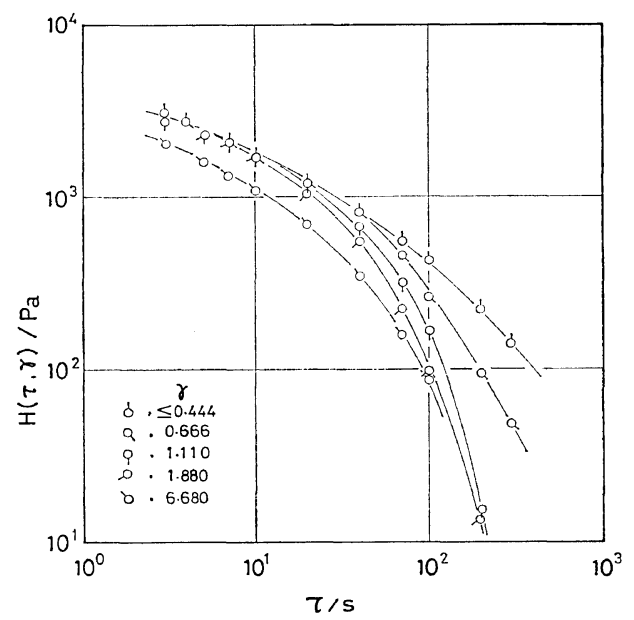

Figure 13. Strain-dependent relaxation spectra evaluated from relaxation moduli of Figure 11 with Ferry-Williams approximation method.

4 is related to the large strain-dependence of the relaxation spectrum at long times. ${ }^{* 2}$ The destruction of the structure formed by the hard domain of a solution of polystyrene blocks may be the origin of the marked decrease in the relaxation strength at long times with increasing shear, increasing temperature, and decreasing concentration.

*2 The quantities $\dot{\gamma}_{c}$ and $\omega_{c}$ were quite close to each other when the complex viscosity and the steady shear viscosity were calculated from eq 11 and 13 with $\alpha \fallingdotseq 0.4$ irrespective of the shape of the relaxation spectrum. Thus, the ratio $\dot{\gamma}_{c} / \omega_{c}$ may be approximated by $0.4 / \alpha$ when eq 11 is applicable. However, we cannot say if the value $\alpha \fallingdotseq 0.4$ has any particular significance or not. 


\section{K. Osaki, B. Kim, and M. Kurata}

Acknowledgment. Bong-Shik Kim is grateful to the Japan Ministry of Education for the grant to foreign students. This study is partly supported by a grant for Scientific Research D-165212 (1976) from the Ministry of Education.

\section{REFERENCES}

1. G. Kraus and J. T. Gruver, J. Appl. Polym. Sci., 11, 2121 (1967).

2. D. R. Paul, J. E. St. Laurence, and J. H. Troell, Polym. Eng. Sci., 2, 70 (1970).

3. G. Kraus and K. W. Rollmann, Angew. Macrom. Chem., I6/I7 (Nr. 199), 271 (1971).

4. G. Kraus, F.E. Naylor, and W. Rollmann, $J$. Polym. Sci., Part A-2, 9, 1839 (1971).

5. T. Kotaka and J. L. White, Trans. Soc. Rheol., 17, 587 (1973).

6. N. Nemoto, K. Okawa, and H. Odani, Bull. Inst. Chem. Res., Kyoto Univ., 51, 118 (1973).

7. C. I. Chung and J. C. Gale, J. Polym. Sci., 14, 1149 (1976).

8. B. S. Kim, K. Osaki, and M. Kurata, J. Soc. Rheol. Jpn., 4, 16 (1976).

9. K. Osaki, B. S. Kim, N. Bessho, and M. Kurata, J. Soc. Rheol. Jpn., 4, 21 (1976).

10. T. Masuda, Y. Matsumoto, T. Matsumoto, and S. Onogi, J. Soc. Rheol. Jpn., 5, 135 (1977).

11. K. Osaki, B. S. Kim, and M. Kurata, Polym. J, 10, 353 (1978).
12. M. Tamura, M. Kurata, K. Osaki, Y. Einaga, and S. Kimura, Bull. Inst. Chem. Res., Kyoto Univ., 49, 43 (1971).

13. See for example, W. W. Graessley, Adv. Polym. Sci., 16, 1 (1974).

14. W. P. Cox and E. H. Merz, J. Polym. Sci., 28, 619 (1958).

15. K. Osaki, M. Fukuda, and M. Kurata, J. Polym. Sci. Polym. Phys. Ed., 13, 775 (1975).

16. M. Fukuda, K. Osaki, and M. Kurata, J. Polym. Sci. Polym. Phys. Ed., 13, 1563 (1975).

17. K. Osaki, M. Fukuda, S. Ohta, B. S. Kim, and M. Kurata, J. Polym. Sci. Polym. Phys. Ed., 13, 1577 (1975).

18. K. Osaki, S. Ohta, M. Fukuda, and M. Kurata, J. Polym. Sci. Polym. Phys. Ed., 14, 1701 (1976).

19. B. Bernstein, E. A. Kearsley, and L. J. Zapas, Trans. Soc. Rheol., 7, 391 (1963).

20. K. Osaki, A. Murai, N. Bessho, and B. S. Kim, J. Soc. Rheol, Jpn., 4, 166 (1976).

21. M. Fukuda, K. Osaki, and M. Kurata, J. Soc. Rheol. Jpn., 3, 114 (1975).

22. See for example, I-J. Chen and D. C. Bogue, Trans. Soc. Rheol., 16, 59 (1972); M. Sakai, H. Fukaya, and M. Nagasawa, ibid., 16, 635 (1972).

23. L. J. Zapas and J. C. Phillips, J. Res. Nat. Bur. Stand., 75A, 33 (1971).

24. M. Yamamoto, "Buttai-no Henkeigaku", Seibundo-Shinkosha, Tokyo, 1972, Chapter 6.

25. J. D. Ferry and M. L. Williams, J. Colloid Sci., 7, 347 (1952). 